Manfred Klenner

Computational Linguistics

University of Zurich \}

Switzerland

klenner@cl.uzh.ch

\title{
An Hymn of an even Deeper Sentiment Analysis
}

A deeper understanding of what is going on in a given text is still one of the most interesting and challenging goals in NLP. Sentiment analysis has recently started to contribute to this area. We no longer just try to predict the polarity of whole product reviews but to distinguish various perspectives inherent to a text, namely, what the author is telling us, how he implicitly or explictely evaluates it and what his text tells us about the attitudes the entities in the text hold towards each other (or towards the mentioned objects, situations, or opinions of others). Other perspectives not yet taken by our systems include the common-sense perspective (what follows from the behaviour of an agent for his perception by the public) or the reader perspective: given that I have specified the pros and cons of my world view - which are the opponents and proponents of mine given the text at hand. Progress has been made in this direction. Sentiment inferences based on verb-specific polar lexicons and general inference rules have been proposed (see the work of Deng and Wiebe, for instance). Our preprocessing tools for the extraction of predicate argument structures or for semantic role labeling seem to be mature enough to support this kind of deep understanding reasonably well.

This is most exciting, like any attempt to reap the benefits of a bit more semantics compared to attemps that just scratch the surface with crude and brute-force means. Sentiment analysis certainly is a field that takes advantage of lexical resources. For instance, verbs have proved to provide a lot of crucial information for fine-grained sentiment analysis taking the various perspectives into account that I mentioned.

Clearly, all the other problems need to be solved as well: aspect detection/induction, sentiment composition, sense disambiguation and so on. New techniques (deep learning), new theoretical stances (distributional semantics) might prove successful. If not, a shift towards a new playgroud is to be expected, e.g. the focus on emotions. Well, I am quite sure that all this will turn out to be very entertaining and - step by step - sucessful. 